High Energy Phenomena in Relativistic Outflows III (HEPRO III)

International Journal of Modern Physics: Conference Series

Vol. 8 (2012) 265-270

(C) World Scientific Publishing Company

DOI: $10.1142 /$ S2010194512004692

\title{
HELICAL MAGNETIC FIELDS IN RELATIVISTIC JETS THROUGH FARADAY ROTATION AND JET STRATIFICATION STUDIES
}

\author{
JOSÉ L. GÓMEZ, CAROLINA CASADIO, MAR ROCA-SOGORB and IVÁN AGUDO \\ Instituto de Astrofísica de Andalucía, CSIC, Apartado 3004, 18080 Granada, Spain \\ jlgomez@iaa.es; iagudo@iaa.es; mroca@iaa.es
}

\author{
ALAN P. MARSCHER and SVETLANA G. JORSTAD \\ Institute for Astrophysical Research, Boston University, \\ 725 Commonwealth Avenue, Boston, MA 02215, USA \\ marscher@bu.edu; jorstad@bu.edu
}

\begin{abstract}
Helical magnetic fields may play an important role in the formation, collimation, and acceleration of relativistic jets in active galactic nuclei. These may be searched for by looking for Faraday rotation measure (RM) gradients and emission stratification across the jet width. Multi-epoch polarimetric Very Long Baseline Array (VLBA) observations of the radio galaxy 3C 120 have revealed the existence of such a RM gradient across the jet, but the presence of a localized region of enhanced RM and uncorrelated changes in the polarization of the underlying jet emission and the Faraday rotation screen suggest that a significant fraction of the RM found in 3C 120 originates in foreground clouds. Thanks to the combination of 48 images spanning 14 years of $15 \mathrm{GHz}$ VLBA observations of $3 \mathrm{C} 273$ we have found a stratification in total intensity across the jet that flips sides with distance along the jet, supporting a model in which the jet of $3 \mathrm{C} 273$ accelerates and is threaded by a helical magnetic field.
\end{abstract}

Keywords: galaxies: active - galaxies: individual (3C 120, 3C 273) - galaxies: jets polarization - radio continuum: galaxies

\section{Introduction}

Recent numerical simulations show that helical magnetic fields may appear naturally through the rotation of the accretion disk or black hole ergosphere from which jets are launched, and could play an important role in the formation of relativistic jets. ${ }^{1}$ The actual collimation and acceleration of the jet takes place in the innermost jet regions, probably within the inner few dozens Schwarzschild radii. ${ }^{2}$ These scales cannot be imaged directly by Very Long Baseline Interferometry (VLBI) observations, but can be probed through multi-wavelength (from radio to $\gamma$-rays) observations, revealing the dynamics, magnetic field structure, and emission mechanisms at high energies. ${ }^{3,4,5,6,7}$ In particular, multi-wavelength observations of BL Lac (Ref. 3), PKS 1510-089 (Ref. 4) and 3C 454.3 (Ref. 5) show that it is common to observe a rapid rotation of the optical polarization angle previous to the ejection of a new 
superluminal component in the VLBI jet. This is interpreted as a result of the motion of the perturbation through a helical magnetic field, therefore providing one of the first observational evidence for the existence of such helical magnetic fields upstream of the radio core, where jets are accelerated and collimated. Beyond the radio core it is also possible to search for helical magnetic fields by looking for Faraday rotation measure gradients and emission stratification across the jet width, as it is discussed in more detail in the following sections.

\section{Gradients in Faraday rotation measure across the jet width: The case of $3 \mathrm{C} 120$}

If jets in active galactic nuclei (AGN) are surrounded by a sheath of nonrelativistic electrons threaded by a helical magnetic field we may expect to observe gradients in Faraday rotation measure (RM) across the jet width. These gradients should appear due to the change in the line-of-sight magnetic field component across the jet. ${ }^{8}$ This represents however an observational challenge, since high angular resolution is required to resolve the jet across its width, and this can only be achieved in a limited number of sources. ${ }^{9}$

One of the sources in which high frequency (15 to $43 \mathrm{GHz}$ ) Very Long Baseline Array (VLBA) observations are capable of resolving the jet across its width is the radio galaxy $3 \mathrm{C} 120 .{ }^{10,11,12,13,14,15}$ This is a very active source in which multiple superluminal components can be used to map the linearly polarized emission, and hence the RM along and across the jet. Furthermore, evidence for the presence of a helical magnetic field in 3C 120 has been found through comparison with numerical simulations. ${ }^{16}$

A sequence of 12 monthly polarimetric 15, 22 and $43 \mathrm{GHz}$ VLBA observations carried out during 2001 has revealed the existence of a stationary RM sheath in 3C $120 .{ }^{17}$ The obtained mean values of the RM and RM-corrected electric vector position angle (EVPA), as well as RM slices across and along the jet are shown in Fig. 1. A transverse stratification in the RM across the jet, as expected in the case that the jet of 3C 120 is threaded by a helical magnetic field, can be observed in Fig. 1. The positive gradient in RM toward the southern side of the jet would require the helical magnetic field to be oriented counterclockwise relative to the flow direction. The longitudinal profile of the RM shown in Fig. 1 reveals a localized region of enhanced rotation measure between $\sim 3-4$ mas from the core, with a peak of $\sim 6000 \mathrm{rad} \mathrm{m}^{-2}$. A smooth sheath around the jet in 3C 120 cannot produce a localized region of enhanced RM, so a local process, such as interaction of the jet with the external medium or a cloud, would be required in order to explain the existence of this region.

In order to study whether most of the observed Faraday rotation in 3C 120 originates in a sheath or foreground clouds we present in Fig. 2 the comparison between the mean RM map of 2001 with the obtained in 1999 January. To quantify any possible variation in the Faraday screen across epochs we have subtracted the 


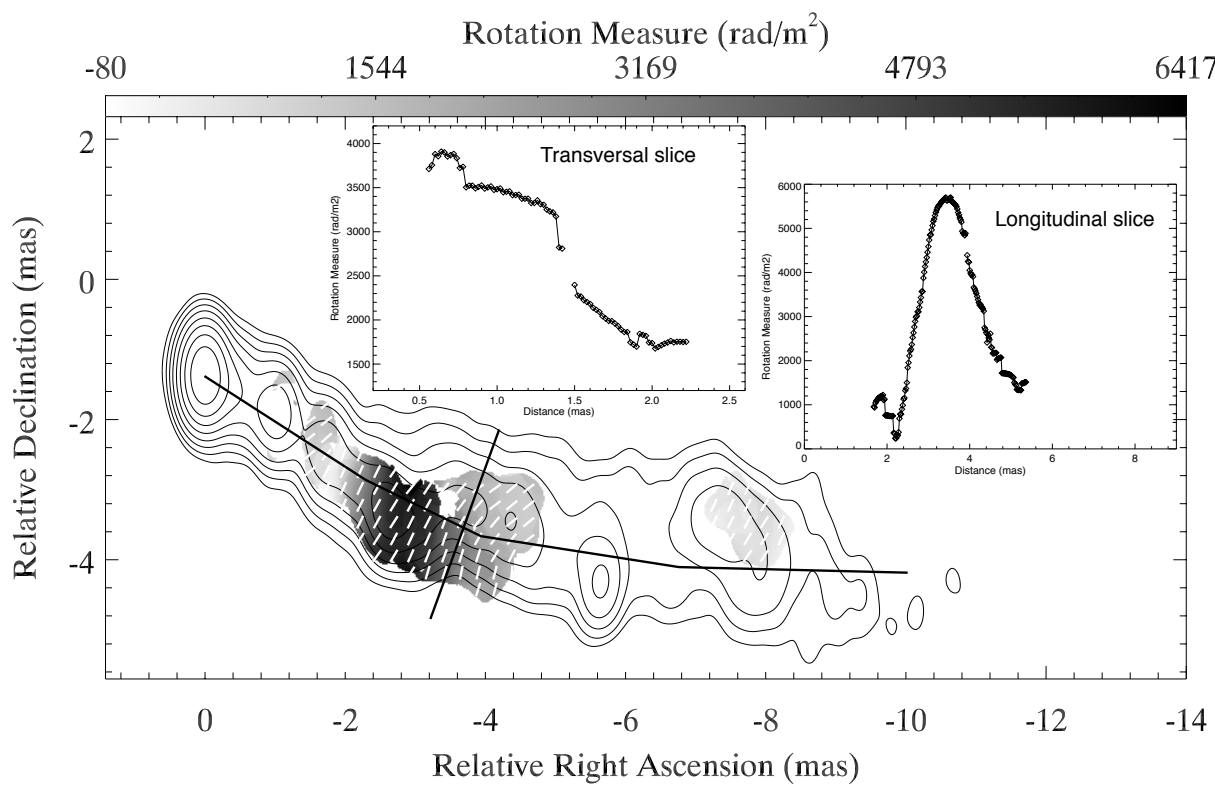

Fig. 1. Map of the mean value of the Faraday rotation measure in 3C 120 obtained from the combination of 12 monthly 15, 22 and $43 \mathrm{GHz}$ VLBA epochs during 2001. Total intensity at 22 $\mathrm{GHz}$ for epoch 2001.35 is shown for reference, with contours overlaid at 1.7, 3.6, 7.7, 16, 35, 74, 157, 334, and $710 \mathrm{mJy} /$ beam. Bars indicate the mean value of the RM-corrected EVPAs. The thick lines indicate the direction of the RM slices shown in the inset panels.

rotation measure values of 1999 January from those of the mean RM map for 2001 and computed the mean value of the difference, as shown in Fig. 2.

We find uncorrelated changes in the linear polarization of the underlying jet emission and the Faraday rotation screen. While the RM remains constant in the outer jet (including the localized region of high rotation measure $\mathrm{S}_{1}$ ) the RMcorrected EVPAs of two particular components $\left(S_{1}\right.$ and $\left.S_{2}\right)$ rotate by almost $90^{\circ}$. On the other hand, in the innermost 2 mas the RM changes significantly but without variations in the RM-corrected EVPAs. These uncorrelated changes suggest that the emitting jet and source of RM are not closely connected physically. Furthermore, the existence of a three-year-long stationary region of enhanced RM requires a localized source of Faraday rotation, which favors a model in which a significant fraction of the Faraday rotation measure found in 3C 120 originates in foreground clouds, rather than in a sheath intimately associated with the emitting jet. ${ }^{17}$ In this case, Faraday rotation studies will provide valuable information about the ambient medium through which jets propagate, but will not be able to reveal further details about the emitting jet, such as the line-of-sight magnetic field, and hence to test whether they are threaded by helical magnetic fields. 


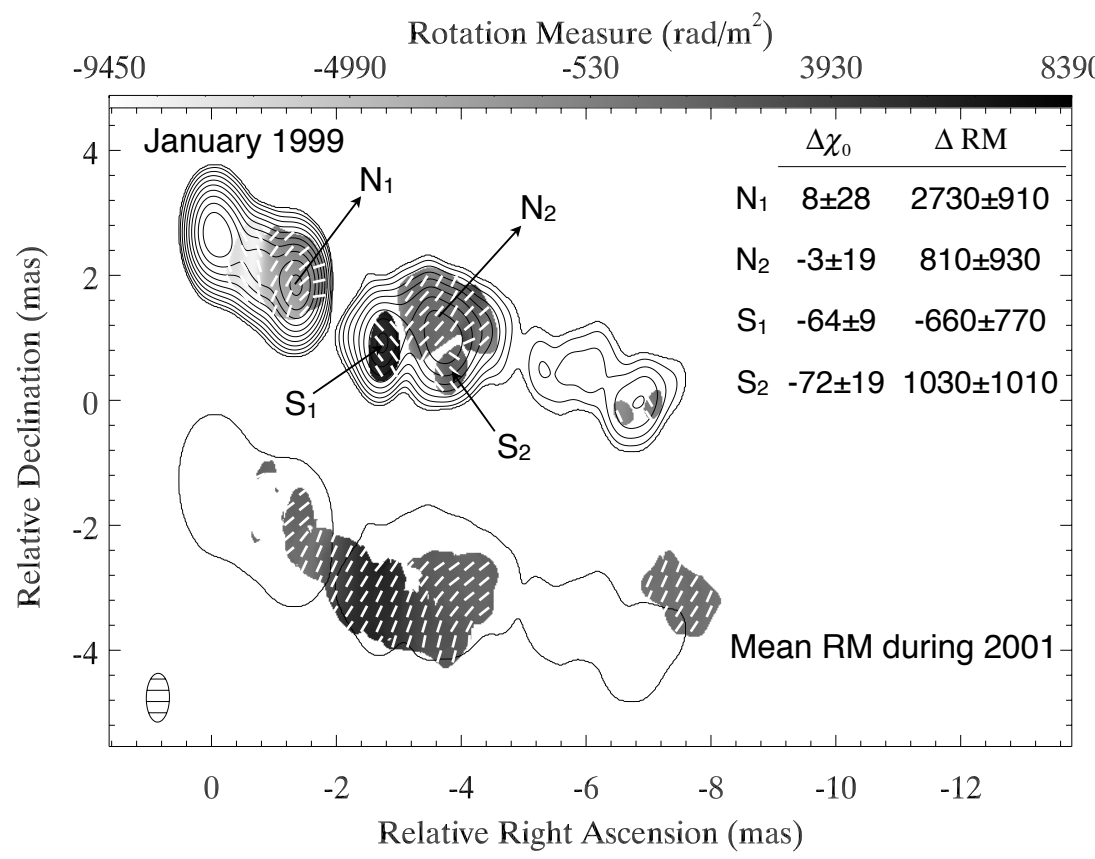

Fig. 2. (top) Rotation measure map of 3C 120 in 10 January 1999 from VLBA observations at 22 and $43 \mathrm{GHz}$. Total intensity $22 \mathrm{GHz}$ contours are overlaid at 3.6, 5.9, 9.8, 16, 27, 44, 73, 120, 198, 327 and $540 \mathrm{mJy} /$ beam. (bottom) Map of the mean value of the rotation measure for observations during 2001 shown for comparison. Bars indicate the RM-corrected EVPAs. The lowest contour of the January 1999 image is also shown for an easier comparison. Inset table shows the mean difference in RM-corrected EVPAs and RM between epochs.

\section{Emission stratification across the jet width: The case of $3 \mathrm{C} 273$}

If the synchrotron emitting jet region is threaded by a helical magnetic field we should expect a stratification in both, total and linearly polarized emission across the jet width. ${ }^{18,19}$ This is produced by the dependence of the synchrotron radiation coefficients on the sinus of the angle between the magnetic field and the line of sight in the fluid frame, $\vartheta$.

Consider a helical magnetic field with a pitch angle $\phi$, measured with respect to the jet axis. The angles $\vartheta^{t}$ and $\vartheta^{b}$ (where superscripts $t$ and $b$ refer to the top and bottom of the jet, respectively) add $2 \phi$ (note that $\vartheta^{t, b}$ is always defined as positive). Therefore, as long as $\phi$ is different from zero or $\pi / 2$, i.e. the field is neither purely aligned nor toroidal, the factor $\sin \vartheta^{t, b}$ in the synchrotron radiation coefficients will introduce an asymmetry in the jet emission. This asymmetry will reach a maximum value for a helical magnetic field with $\phi=\pi / 4$. However, independently of the helix pitch angle, the predominance between $\sin \vartheta^{t}$ and $\sin \vartheta^{b}$ will reverse at $\theta^{\prime}=\pi / 2$, which corresponds to a viewing angle in the observer's frame of $\cos \theta_{r}=\beta$. It is interesting to note that for $\theta \sim \theta_{r}$, small changes in the jet velocity or the viewing angle will produce a flip in the top/bottom jet emission dominance. 


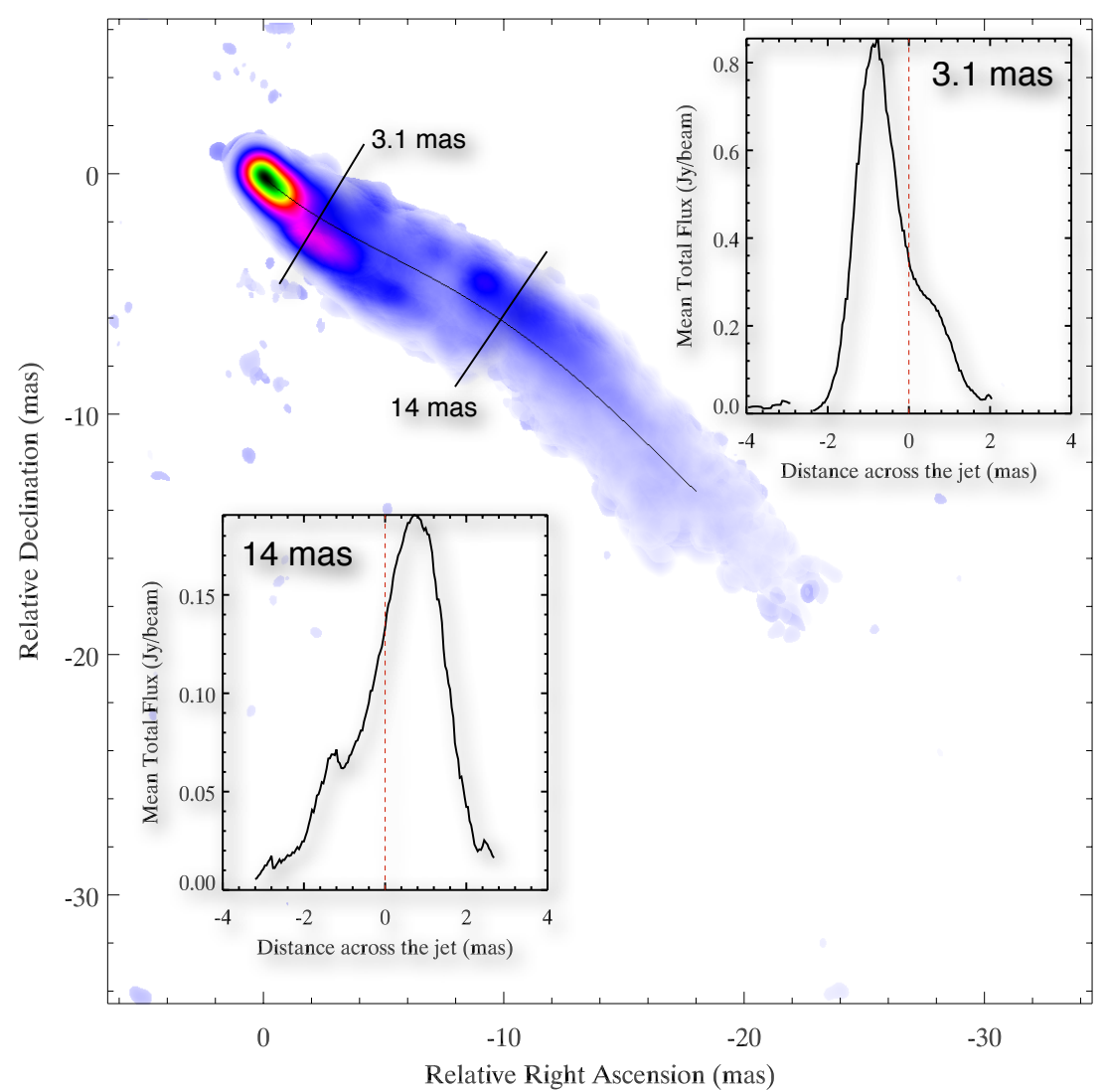

Fig. 3. Total intensity mean value of $3 \mathrm{C} 273$ obtained by combining information corresponding to 48 epochs spanning 14 years of VLBA observations at $15 \mathrm{GHz}$, including data from the MOJAVE program. Inset panels show the slices at 3.1 and 14 mas from the core. A black line extending from the core indicates the estimated jet ridge-line.

A source in which we may expect to see an asymmetry in the emission is $3 \mathrm{C} 273$, since it shows one of the best-established cases of transverse RM gradients. ${ }^{20,21,22}$ To search for emission stratification across the jet in 3C 273 we have combined the information corresponding to 48 epochs spanning 14 years of VLBA observations at $15 \mathrm{GHz}$, including data from the MOJAVE program. The obtained mean value of the total intensity and slices across the jet are shown in Fig. 3. As expected for the case of a helical magnetic field, we find a stratification in emission across the jet. Most interestingly, the side of the jet that is brighter changes with distance from the core. As mentioned previously, this can be explained by an acceleration of the jet flow with distance along the jet, as it has been measured in several superluminal components. ${ }^{23,24}$ We therefore conclude that both, the stratification in RM and emission across the jet width, support a model in which the jet of $3 \mathrm{C} 273$ accelerates with distance from the core and is threaded by a helical magnetic field. 


\section{Acknowledgements}

This research has been supported by the Spanish Ministry of Science and Innovation grant AYA2010-14844, by the Regional Government of Andalucía (Spain) grant P09-FQM-4784, and by National Science Foundation grant AST-0907893. This research has made use of data from the MOJAVE database that is maintained by the MOJAVE team (Lister et al. 2009, AJ, 137, 3718). The VLBA is an instrument of the National Radio Astronomy Observatory, a facility of the National Science Foundation operated under cooperative agreement by Associated Universities, Inc.

\section{References}

1. J. C. McKinney and R. D. Blandford, Mon. Not. R. Astron. Soc. 394, L126 (2009).

2. K. Hada, et al., Nature 477, 185 (2011).

3. A. P. Marscher et al., Nature 452, 966 (2008).

4. A. P. Marscher, et al., Astrophys. J. Lett. 710, L126 (2010).

5. S. G. Jorstad, et al., Astrophys. J. 715, 362 (2010).

6. I. Agudo, et al., Astrophys. J. Lett. 726, L13 (2011).

7. I. Agudo, et al., Astrophys. J. Lett. 735, L10 (2011).

8. R. D. Blandford, in Astrophysical Jets, eds. D. Burgarella, M. Livio and C. P. O'Dea (Cambridge Univ. Press, Cambridge, 1993), p. 15.

9. G. B. Taylor and R. Zavala Astrophys. J. Lett. 722, L183 (2010).

10. J. L. Gómez, et al., Astrophys. J. 499, 221 (1998).

11. J. L. Gómez, A. P. Marscher and A. Alberdi Astrophys. J. Lett. 521, L29 (1999).

12. J. L. Gómez, et al., Science 289, 2317 (2000).

13. J. L. Gómez, et al., Astrophys. J. Lett. 561, L161 (2001).

14. A. P. Marscher, et al., Astrophys. J. 665, 232 (2007).

15. M. Roca-Sogorb, et al., Astrophys. J. Lett. 712, L160 (2010).

16. P. R. Hardee, R. C. Walker and J. L. Gómez Astrophys. J. 620, 646 (2005).

17. J. L. Gómez, et al., Astrophys. J. Lett. 681, L69 (2008).

18. R. A. Laing, Astrophys. J. 248, 87 (1981).

19. M. A. Aloy, et al., Astrophys. J. 528, L85 (2000).

20. K. Asada, et al., in Pub. Astron. Soc. Japan 54, L39 (2002).

21. K. Asada, et al., Astrophys. J. 675, 79 (2008).

22. R. T. Zavala and G. B. Taylor, Astrophys. J. 626, L73 (2005).

23. S. G. Jorstad, et al., Astron. J. 130, 1418 (2005).

24. M. L. Lister, et al., Astron. J. 138, 1874 (2009). 